\title{
Experimental Validation of a CFD-based Air Quality Sensor Placement Strategy for the Localization of Indoor Source Emissions
}

\author{
Julien Waeytens ${ }^{1,2}$, Sophie Durand ${ }^{1}$, Sara Sadr ${ }^{1}$ \\ ${ }^{1}$ Université Paris-Est, IFSTTAR, Marne-la-Vallée, France \\ ${ }^{2}$ Efficacity, Marne-la-Vallée, France
}

\begin{abstract}
Some woodborers such as termites have harmful effects on the safety of structures. As the woodborers produce some specific Volatile Organic Compounds (VOC), they can be used as an indicator to early detect their presence. Thus, innovative solutions combining gas sensors outputs and Computational Fluid Dynamics (CFD) are studied to localize indoor source emissions. These strategies can also be applied to localize noxious indoor pollutants like formaldehyde. In this paper, we focus on the optimal placement of air quality sensors in view of localizing a maximum of source emissions on the environment surfaces. We present an adjoint-based numerical strategy taking into account the sensor features such as the Limit Of Detection (LOD). The main contribution of this article is the application of the optimal sensor placement strategy to a real 3D room and its validation by an experimental campaign.
\end{abstract}

\section{Introduction}

As people spend approximately $80 \%$ of their time in indoor environments, increasing attention has been focused on indoor air quality (IAQ). Volatile organic compounds (VOCs) are characteristic chemical species present in indoor environments. Several studies have shown that the concentration of VOCs can be higher in indoor locations compared to the concentrations outside (Hoang et al. (2017); Godwin and Batterman (2006); Goodman et al. (2018); Campagnolo et al. (2017); Bari et al. (2015)). As permanent and occasional exposure, even at low VOC levels, has an impact on human health (Organization (2010)), it is important to monitor indoor air quality and to precisely localize sources to propose an appropriate action plan to improve air quality. The monitoring of air quality is facilitated by the improvement in sensor technologies, notably nanotechnologies. Hence, the gas sensors become cheaper, smaller, more sensitive, less energyconsuming, etc... The localization of VOC sources can also be useful for the preservation of cultural heritage, notably artwork, and for structural health monitoring purposes. In most regions of France, the presence of woodborers, such as termites, has harmful effects on the safety of structures. The VOC chemical signature of termites can be used for their early detection and localization, which will provide the ability to limit the use of termiticides and to preserve the structure.

To efficiently monitor air quality, the number of sensors and their positioning are crucial. In most measurement campaigns, the gas sensors are placed in an empirical way. For example, in a room, an air quality sensor is usually positioned at the breathing zone height or approximately $0.5 \mathrm{~m}$ from the ceiling in the middle of the room. Unfortunately, this placement does not take into account the characteristics of the room, i.e. the geometry and the ventilation. As a consequence, bad sensor placement may lead to the nondetection of some sources. To well-position gas sensors, we can take advantage of numerical simulations derived from physical models. In indoor air quality applications, the gas concentration can be predicted using multizone (Bourdin et al. (2014); Dimitroulopoulou et al. (2006); Haghighat et al. (1988); Nazaroff and G. (1986)) and CFD (Bourdin et al. (2014); Yan et al. (2009); Gan and Awbi (1994)) models. Multizone techniques, which provide the time evolution of the averaged concentration in each zone as output, are easy to use and run on a standard laptop. Nevertheless, they consider strong hypotheses, such as a well-mixed concentration. With the ongoing improvement of computers and numerical methods, CFD approaches appear to be promising for the prediction of indoor air quality and for optimal sensor placement. In fact, CFD provides a fine description of the spatial concentration in the indoor environment, but the computations are time consuming. To the best of the authors' knowledge, few publications have addressed the optimal placement of gas sensors for IAQ applications. The design 
of an optimal sensor network, i.e. the number and positioning of sensors, has been studied in greater depth in terms of chemical and biological warfare (CBW) and transmission of infectious diseases (TID). The sensor positions are chosen to early detect and localize indoor contamination. Different methods aim to maximize the coverage area of sensors and to minimize the response time for various sets of release scenarios. In (Liu and Zhai (2009)), the sensor coverage area is evaluated using CFD and an adjoint advection-diffusion equation, whereas physical model-free approaches based on a dynamical systems approach are preferred in (Fontanini et al. (2016)).

Once the positions of the sensors are fixed, the knowledge of the concentration given by the deployed sensors is not sufficient for proposing efficient solutions for indoor air quality improvement or for localizing woodborers. One needs to localize and to quantify the source emissions. To achieve this purpose, two families of methods can be found in the literature, i.e. data-driven methods and physical model-based methods. Direct measurements of the source emissions on different surfaces of the environment (furniture, wall, floor, door, etc.) can be planned using innovative sensors, such as fibers placed in a specific device for on-site emission control (Desauziers et al. (2015); Bourdin et al. (2014)). This method enables accurate in-situ quantification of the source emissions for building materials and furniture, but it requires a large number of sensor devices. Another data-driven method to evaluate source emissions is indirect measurements. In contrast to the previous methods, the air quality sensors are placed in the room volume and not directly on a surface. Databases of the chemical signatures of sources and a priori information of the studied environment collected via questionnaire, including the type and the age of the building materials, renovations, cleaning products and ventilation, are commonly considered in these methods. Finally, the sensor outputs associated with various chemical compounds are analyzed via statistical tools, such as proper component analysis and linear regression, to identify the source emissions (Campagnolo et al. (2017); Bari et al. (2015); Wang et al. (2014); Clarisse et al. (2003)). In practice, the chemical compounds emitted by some items in the studied environment may not be referenced in a database. Consequently, these methods may only approximately identify the sources. Physical model-based approaches via inverse modeling techniques can also be valuable for the localization and the quantification of source emissions but require a sufficient number of well-positioned sensors.

In (Waeytens and Sadr (2018)), we propose a virtual testing strategy, taking into account the specificities of the indoor environment (geometry and ventilation) via CFD and gas sensor features (limit of detection), to efficiently select the number and positions of sensors to localize indoor sources. We define the "optimal sensor placement" as the combination of gas sensors that maximizes the coverage area. Herein, we emphasize that the coverage area can be increased not only by adding sensors but also by using sensors with a lower limit of detection. The main novelty in this proceeding concerns the validation of this numerical strategy by an experimental campaign conducted in a real room. It consists to inject a chemical compound at a predefined location, which corresponds to the source, and to measure the gas concentration at the optimal sensor positions. We show that the gas sensor, which covers the source area, correctly detects the injected gas.

\section{Ajoint-based Numerical Method for Optimal Sensor Placement}

Before presenting the numerical strategy for the optimal placement of gas sensors, let us define the adjoint equations (1) which corresponds herein to a backward-advection-diffusion problem with a source emission $f_{s}$ located at a given sensor position $\mathbf{x}_{\mathbf{s}}$.

$$
\left\{\begin{array}{l}
-\mathbf{v}(\mathbf{x}) \cdot \nabla \tilde{C}(\mathbf{x})-\nu(\mathbf{x}) \Delta \tilde{C}(\mathbf{x})=f_{s}\left(\mathbf{x}-\mathbf{x}_{\mathbf{s}}\right) \text { in } \Omega \\
\tilde{C}(\mathbf{x})=0 \quad \text { on } \partial_{p} \Omega \\
\tilde{C}(\mathbf{x})=0 \quad \text { on } \partial_{u} \Omega \\
\nabla \tilde{C}(\mathbf{x}) \cdot \mathbf{n}=0 \quad \text { on } \partial_{n} \Omega \\
\nu \nabla \tilde{C}(\mathbf{x}) \cdot \mathbf{n}+\mathbf{v}(\mathbf{x}) \cdot \mathbf{n} \tilde{C}(\mathbf{x})=0 \quad \text { on } \partial_{o} \Omega
\end{array}\right.
$$

In Eq. (1), $\tilde{C}$ denotes the adjoint concentration field, $\mathbf{v}$ is the velocity field obtained from CFD simulations and $\nu$ is the sum of the molecular and the turbulent diffusivity. Four types of boundaries can be distinguished. A boundary presenting a known prescribed concentration $C_{p}$ is denoted $\partial_{p} \Omega$. Potential pollution emissions, to be precisely located by the optimal placement of gas sensors, are on the boundary $\partial_{u} \Omega$, whereas a boundary that does not present source emission is $\partial_{n} \Omega$. Lastly, $\partial_{o} \Omega$ denotes the outgoing flow boundary.

The number and the position of gas sensors are selected in view of maximizing the coverage area and thus detecting a maximum of sources on the surfaces. For a sensor located at a given position $\mathbf{x}_{\mathbf{s}}$, its coverage area is estimated numerically using the following criterion introduced in (Waeytens and Sadr (2018)):

$$
\mathbf{x} \in \partial_{u} \Omega \text { such that }|\nu(\mathbf{x}) \nabla \tilde{C}(\mathbf{x}) \cdot \mathbf{n}| \frac{A_{s} S}{d I_{m}}>1 \text {. }
$$

where:

- $\tilde{C}$ is the adjoint concentration field computed by solving Eq. (1) 
- $A_{s}$ is the minimum source area expected to be localized

- $S$ is the order of magnitude of the source emission

- $d I_{m}$ is the limit of detection of the gas sensor

In the optimal sensor placement strategy, we first list all potential sensor positions and we evaluate their coverage area using the criterion (2). The gas sensor having the highest coverage area is selected as optimal. The first optimal sensor position is fixed and the second sensor position is sought to maximize the coverage area. The numerical method stops when the maximum number of sensors or the expected coverage area are reached.

\section{Presentation of the case study and op- timal sensor placement}

To validate the computer-aided method for the optimal placement of gas sensors, we consider a real 3D laboratory room (see Figure 1), including furniture and ventilation systems, located at the IFSTTAR research institute. The dimensions of the room are $5.9 m \times 6.2 m \times 4.2 m$, which correspond to a volume of $150 \mathrm{~m}^{3}$. The flow incomes into the room from the heating duct, the two ventilation grids and the door whereas it exits only from the extractor hood. From the numerical mock-up, we simulate the stationary turbulent flow using the $k-\omega S S T$ Reynolds Average Navier-Stokes (RANS) model in the CFD software "Code_Saturne" (Archambeau et al. (2004)). To impose representative CFD boundary conditions, the incoming flows were measured using a $1 \mathrm{D}$ hot wire anemometer. The values are reported in Figure 1. We can see in this figure that the airflow entering from the contour of the door is highly turbulent in the vicinity of the door and that a portion of it goes straight in the direction of the extractor hood. In terms of the incoming flow from the second ventilation grid, the main portion circulates close to the ground between the wall and the furniture. Lastly, the velocity flow from the heating duct oriented in the z-direction impacts the top of the furniture immediately below, which generates flow recirculation. To check the numerical flow, it was compared in previous works with few pointwise measured velocities using a 3D ultrasonic anemometer.

In the laboratory room, the optimal sensor positions are sought from a set of 363 potential sensor positions equally distributed every $50 \mathrm{~cm}$ at three heights above the ground, namely, $0.5 \mathrm{~m}, 1 \mathrm{~m}$ and $1.5 \mathrm{~m}$. The goal is to select the sensors maximizing the coverage area on all the lateral surfaces (door face, furniture face, extractor hood face, back face) defined in Figure 2. To quantify the coverage area associated to each potential sensor positions, we use the observable criterion (2), taking into account the sensor features and source characteristics.

In the experimental campaign, we aim to reproduce
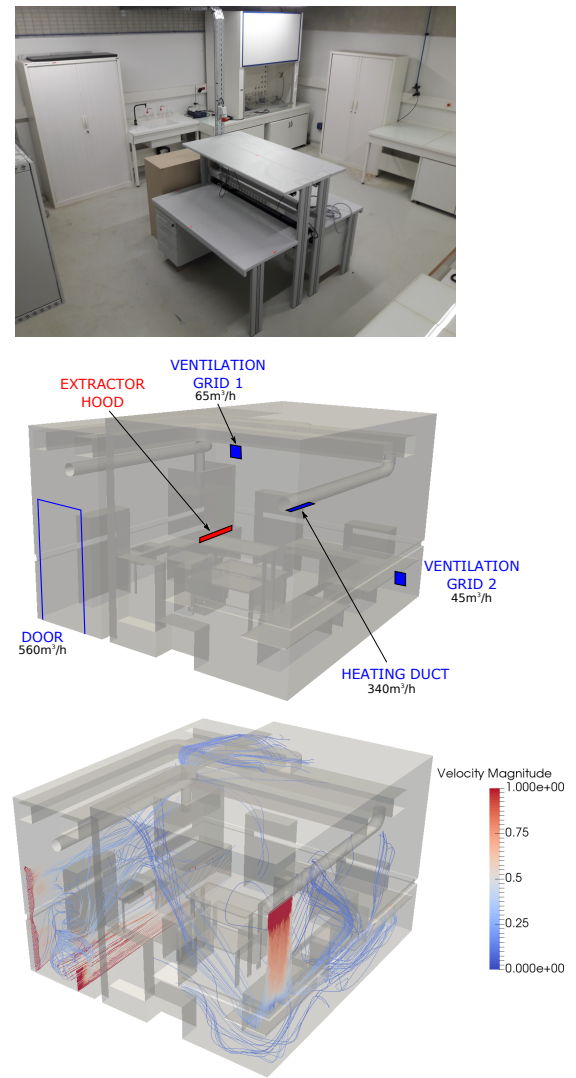

Figure 1: Picture of the laboratory room, Numerical mock-up with measured incoming flows and Flow simulated by CFD software.

a source emission and to verify that it is properly detected and localized thanks to the optimally placed sensors. For convenience, we retain a CO2 source emission at $10000 \mathrm{ppm}$ obtained from a reference gas cylinder. The CO2 reference gas cylinder is linked using a tube to a source emission device of $0.25 \mathrm{~m}^{2}$ which is represented in Figure 4. Concerning the measurement of CO2 concentration, we use Nondispersive Infrared (NDIR) sensors. The measurement error of these gas sensors at ambient air $\mathrm{CO} 2$ concentration is about 50ppm. Thus, considering

$$
A_{s}=0.25 m^{2}, S=10000 p p m \text { and } d I_{m}=50 p p m
$$

in the criterion (2), the CFD-based strategy selects the 4 sensor positions represented in Figure 2.

In Figure 2, the sector colors indicates the faces observable by the sensor. Both S2 and S3 are sensitive to gas emission on the furniture face whereas $\mathrm{S} 1$ (resp. S4) mostly covers the back face (resp. the extractor hood face). For the 4 optimal sensor positions, the detail of the coverage areas is represented in Figure 3. We can see that $\mathrm{S} 2$ is able to detect a source in the lower part of the furniture face while S3 covers a small area in the upper part of this wall. 


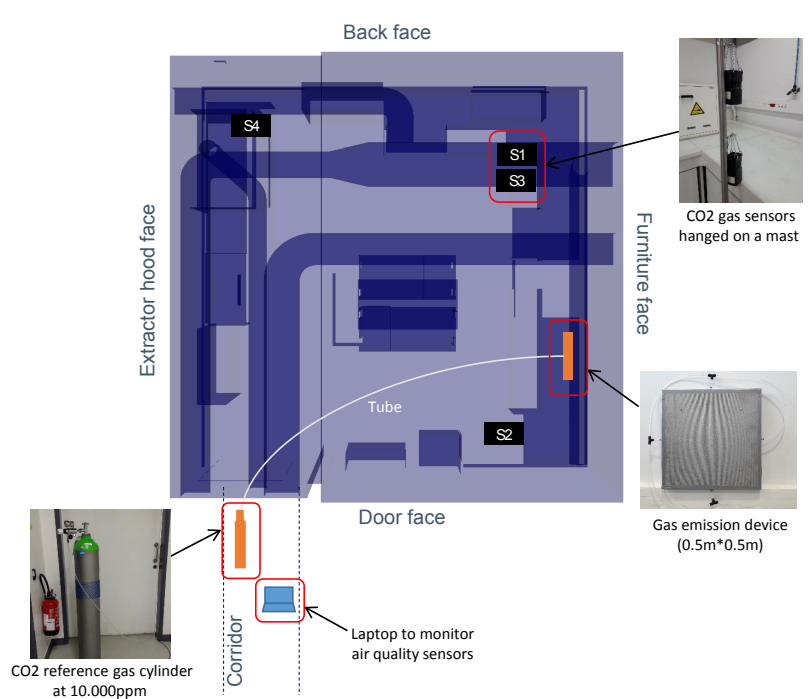

Figure 4: Overview of the CO2 gas emission experimental campaign in the laboratory room.

tions, i.e. 500ppm and 2000ppm, and a concentration verification point at $1000 \mathrm{ppm}$. Then, the CO2 gas sensors $\mathrm{S} 1, \mathrm{~S} 2, \mathrm{~S} 3$ and $\mathrm{S} 4$ are placed at the positions obtained from the CFD-base method. The background $\mathrm{CO} 2$ concentrations are registered with a time step of $10 \mathrm{~min}$ during two hours in controlled conditions, i.e. no occupancy and closed door. The mean values of the $\mathrm{CO} 2$ background concentration at the sensor locations on two hours are given in Figure 5 and noted "B". Then, we start injecting CO2 gas at $10000 \mathrm{ppm}$ using the CO2 gas cylinder placed in the corridor (outside the room) and the gas emission device. This device has a squared shape of $0.5 \mathrm{~m}$ by $0.5 \mathrm{~m}$ and a thickness of $5 \mathrm{~cm}$. The CO2 is distributed into the device via their fourth lateral faces and it goes out from the front face constituted by a perforated plate R0.5 T1.09. The perforated plate allows to diffuse the $\mathrm{CO} 2$ on a squared area of $0.5 \mathrm{~m}$ by $0.5 \mathrm{~m}$ which aims to reproduce a local source emission. Two hours after the beginning of the gas emission, we have monitored the $\mathrm{CO} 2$ concentration for one hour with a time step of $10 \mathrm{~min}$. The mean values of the concentration during this hour at the sensor locations are reported in Figure 5 and noted "I". Recalling that the measurement error of the considered gas sensors at ambient air CO2 concentration is about 50ppm, we consider that a minimum variation of $50 \mathrm{ppm}$ between the background concentration and the concentration measured during the gas injection stage is required to detect and to localize a source. We observe that only the gas sensor $S_{2}$ fulfills this condition. Hence, according to the CFD-based approach, a source emission may be localized in the coverage area of the sensor $S_{2}$, represented in red in Figure 3. Indeed, the predicted source localization information is correct recalling that the gas emission device was placed in the coverage area of sensor $S_{2}$ (see the white rectangu- 
lar in Figure 3). This constitutes a first experimental validation of the proposed CFD-based to optimally placed gas sensor and to localize indoor source emission.

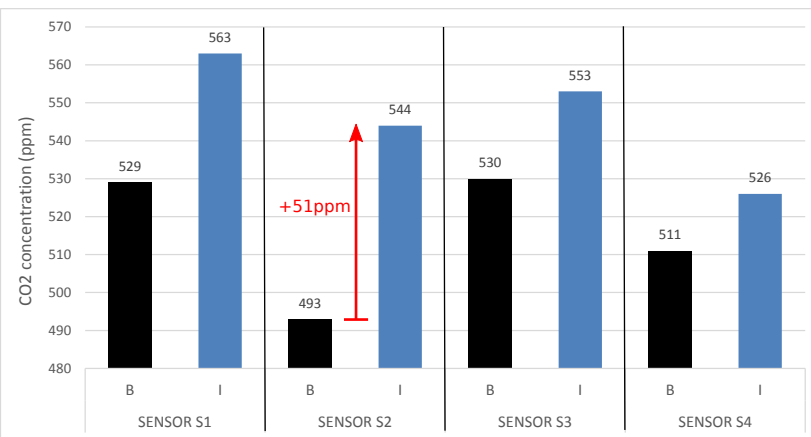

Figure 5: Background CO2 concentrations, noted "B", and CO2 concentrations measured at the optimal sensor locations $S_{1}, S_{2}, S_{3}$ and $S_{4}$ after the injection of gaz into the source emission device, noted "I".

\section{Conclusion}

A CFD-based approach using adjoint framework has been proposed to optimally placed gas sensor. The objective is to place the sensors in view of maximizing the coverage area and of detecting a maximum of indoor source emissions. The numerical technique is applied to a real laboratory room under controlled flow conditions. A first experimental campaign has been conducted to validate the proposed numerical strategy. It consists in reproducing a local indoor source emission using a reference gas cylinder and a squared-shaped gas emission device and in monitoring the gas concentration at the optimal sensor positions. The gas emission device, which reproduces a local source emission, is properly localized by the numerical technique which gives first promising results.

\section{Acknowledgments}

This work was supported by the FUI 18 MIMESYS funded by Region Ile-de-France, which involves several partners: EcologicSense, TERA, ETHERA, FLUIDYN, CSTB, ESIEE Paris, and IFSTTAR. We also want to thank our colleagues Erick Merliot for the realization of the numerical mock-up of the studied room and Rachida Chakir for the fruitful discussions about the direct simulations of the air flow and gas dispersion.

\section{References}

Archambeau, F., N. Méchitoua, and M. Sakiz (2004). Code Saturne: A Finite Volume Code for the computation of turbulent incompressible flows - Industrial Applications. Int. J. on Finite Vol. 1(1).

Bari, A., W. Kindzierski, A. Wheeler, M.-E. Héroux, and L. Wallace (2015). Source apportionment of indoor and outdoor volatile organic compounds at homes in edmonton, canada. Build. and Environ. 90, $114-124$.

Bourdin, D., P. Mocho, V. Desauziers, and H. Plaisance (2014). Formaldehyde emission behavior of building materials: On-site measurements and modeling approach to predict indoor air pollution. J. of Hazard. Mater. 280, $164-173$.

Campagnolo, D., D. E. Saraga, A. Cattaneo, A. Spinazzè, C. Mandin, R. Mabilia, E. Perreca, I. Sakellaris, N. Canha, V. G. Mihucz, T. Szigeti, G. Ventura, J. Madureira, E. de Oliveira Fernandes, Y. de Kluizenaar, E. Cornelissen, O. Hänninen, P. Carrer, P. Wolkoff, D. M. Cavallo, and J. G. Bartzis (2017). Vocs and aldehydes source identification in european office buildings - the officair study. Build. and Environ. 115, $18-24$.

Clarisse, B., A. Laurent, N. Seta, Y. L. Moullec, A. E. Hasnaoui, and I. Momas (2003). Indoor aldehydes: measurement of contamination levels and identification of their determinants in paris dwellings. Environ. Res. 92(3), $245-253$.

Desauziers, V., D. Bourdin, P. Mocho, and H. Plaisance (2015). Innovative tools and modeling methodology for impact prediction and assessment of the contribution of materials on indoor air quality. Herit. Sci. 3(1), 28.

Dimitroulopoulou, C., M. Ashmore, M. Hill, M. Byrne, and R. Kinnersley (2006). Indair: A probabilistic model of indoor air pollution in $\mathrm{uk}$ homes. Atmos. Environ. 40(33), 6362 - 6379.

Fontanini, A. D., U. Vaidya, and B. Ganapathysubramanian (2016). A methodology for optimal placement of sensors in enclosed environments: A dynamical systems approach. Build. and Environ. $100,145-161$.

Gan, G. and H. B. Awbi (1994). Numerical simulation of the indoor environment. Build. and Environ. 29(4), $449-459$.

Godwin, C. and S. Batterman (2006). Indoor air quality in michigan schools. Indoor Air 17(2), 109-121.

Goodman, N., A. Wheeler, P. Paevere, P. Selleck, M. Cheng, and A. Steinemann (2018). Indoor volatile organic compounds at an australian university. Build. and Environ. 135, $344-351$.

Haghighat, F., P. Fazio, and T. Unny (1988). A predictive stochastic model for indoor air quality. Build. and Environ. 23(3), 195 - 201.

Hoang, T., R. Castorina, F. Gaspar, R. Maddalena, P. Jenkins, Q. Zhang, T. McKone, E. Benfenati, 
A. Shi, and A. Bradman (2017). Voc exposures in california early childhood education environments. Indoor Air 27(3), 609-621.

Liu, X. and Z. Zhai (2009). Protecting a whole building from critical indoor contamination with optimal sensor network design and source identification methods. Build. and Environ. 44 (11), 2276 - 2283.

Nazaroff, W. and C. G. (1986). Mathematical modeling of chemically reactive pollutants in indoor air. Environ. Sci. E Technol. 20(9), $924-934$.

WHO Regional Office for Europe (2010). Selected Pollutants.

Waeytens, J. and S. Sadr (2018). Computer-aided placement of air quality sensors using adjoint framework and sensor features to localize indoor source emission. Building and Environment 144, $184-193$.

Wang, C., X. Yang, J. Guan, Z. Li, and K. Gao (2014). Source apportionment of volatile organic compounds (vocs) in aircraft cabins. Build. and Environ. 81, 1-6.

Yan, W., Y. Zhang, Y. Sun, and D. Li (2009). Experimental and cfd study of unsteady airborne pollutant transport within an aircraft cabin mock-up. Build. and Environ. 44(1), 34-43. 